\title{
Lipid droplets of protozoan parasites: survival and pathogenicity
}

\author{
Victor de Souza Tavares ${ }^{1}$, Monara Viera de Castro', Rayane da Silva Oliveira Souza', \\ Iana Kátia Araújo Gonçalves', Jonilson Berlink Limaa,2, Valéria de Matos Borges², \\ Théo Araújo-Santos ${ }^{1,2} /+$ \\ ${ }^{1}$ Universidade Federal do Oeste da Bahia, Centro das Ciências Biológicas e da Saúde, Núcleo de Estudos de Agentes Infecciosos \\ e Vetores, Barreiras, BA, Brasil \\ ${ }^{2}$ Fundação Oswaldo Cruz-Fiocruz, Instituto Gonçalo Moniz, Laboratório de Inflamação e Biomarcadores, Salvador, BA, Brasil
}

Lipid droplets (LDs; lipid bodies) are intracellular sites of lipid storage and metabolism present in all cell types. Eukaryotic LDs are involved in eicosanoid production during several inflammatory conditions, including infection by protozoan parasites. In parasites, LDs play a role in the acquisition of cholesterol and other neutral lipids from the host. The number of LDs increases during parasite differentiation, and the biogenesis of these organelles use specific signaling pathways involving protein kinases. In addition, LDs are important in cellular protection against lipotoxicity. Recently, these organelles have been implicated in eicosanoid and specialised lipid metabolism. In this article, we revise the main functions of protozoan parasite LDs and discuss future directions in the comprehension of these organelles in the context of pathogen virulence.

Key words: lipid droplet - lipid bodies - protozoan - parasites - oxidative stress - lipid metabolism - eicosanoid

\section{Biogenesis of lipid droplets in protozoan parasites}

Most cells present lipid droplets (LDs), also called lipid bodies, which are cytoplasmic organelles involved in lipid compartmentalisation and signaling. All LDs, regardless of cell type, have a similar molecular composition: a hydrophobic neutral lipid core coated by a monolayer of phospholipids. ${ }^{(1)}$ Despite the poorly understood cellular and molecular mechanisms of LD biogenesis in protozoan parasites, knowledge of mammalian cells can shed light on cellular mechanisms and protein involvement in LD formation. ${ }^{(2)}$ More than just an accumulation of neutral lipids, the majority of eukaryotic cells LDs remain stable due to structural proteins such as perilipin, adipose differentiation-related protein (ADRP) and tailinteracting protein of $47 \mathrm{kDa}$ (TIP47). These comprise PAT proteins; they support fatty acid (FA), triacylglycerol (TAG) and cholesteryl ester (CE) uptake and are crucial to LD formation de novo in mammalian cells. . $^{(2,3,4,5)}$ Although none of the genes related to PAT protein production have been described in parasites genomes, the LD kinase (LDK) found in trypanosomatids is the only protein in parasitic protozoa known to be responsible for performing a similar function. ${ }^{(6)}$ Moreover, other organelles take part from the origin until the LD turnover. ${ }^{(7)}$ The endoplasmic reticulum (ER) provides the structural molecular components necessary for biogenesis, and mitochondria capture the molecules mobilised in LDs for use in metabolic functions ${ }^{(8)}$ (Figure and Table I).

\footnotetext{
doi: 10.1590/0074-02760210270

Financial support: CNPq (grant number \#422696/2016-1), FAPESB.

+ Corresponding author: theo.santos@ufob.edu.br

(1) https://orcid.org/0000-0001-9861-6660

Received 11 August 2021

Accepted 14 December 2021
}

Several protozoan pathogens take advantage of lipid metabolite sequestration in the host. Toxoplasma gondii and Plasmodium falciparum display several proteins related to the accumulation of CEs in LDs. ${ }^{(9,10,11,12,13)}$ Acyl-CoA: cholesterol acyltransferase (ACAT)-related enzymes TgACAT and TgACAT2, and acyl-CoA: diacylglycerol acyltransferase (DGAT)-related enzyme TgDGAT, which are responsible for metabolising and synthesising CEs and TAGs contributing to lipid storage in the LDs of these parasites..$^{(9,10,11,12,13)}$ Moreover, $T$. gondii genome encodes a single lipin, TgLIPIN, which is responsible for converting phosphatidic acid to diacylglycerol and phosphate. ${ }^{(14)} T$. gondii knockouts of $T g D G A T$ and $\operatorname{TgLIPIN}$ decrease LD formation..$^{(10,14)}$ suggesting that these genes play a central role in LD biogenesis in this parasite. The physical proximity of protozoan LDs to the ER and mitochondria, ${ }^{(15)}$ suggests that LD biogenesis is both functionally and morphologically dependent on intracellular organelle interactions. Especially during nutrient starvation, the trafficking of FAs from the LDs to into the mitochondria protects cells against the lipotoxicity of autophagic processes. ${ }^{(14,16)}$ The depletion of the Trypanosoma brucei lipin homolog (TbLpn) decreases the number of LDs, simultaneously affecting the structural integrity of the mitochondria and dramatically reducing ATP production, ${ }^{(17)}$ suggesting a role of protozoan LDs in lipid traffic between the ER and mitochondria in parasites (Figure and Table I).

In trypanosomatids, a protein kinase localised intracellularly in close association with the ER and the LD hemi-membrane surface produced insights into the mechanisms responsible for signaling pathways of LD biogenesis in protozoans. Named lipid droplet kinase (LDK) due to a protein kinase domain and LD association, this protein is critical for LD formation. LDKs are activated by autophosphorylation, and induce LD biogenesis in T. brucei. ${ }^{(6)}$ More studies are necessary to elucidate kinases and signaling pathways involved in LD biogenesis in other protozoan pathogens. 


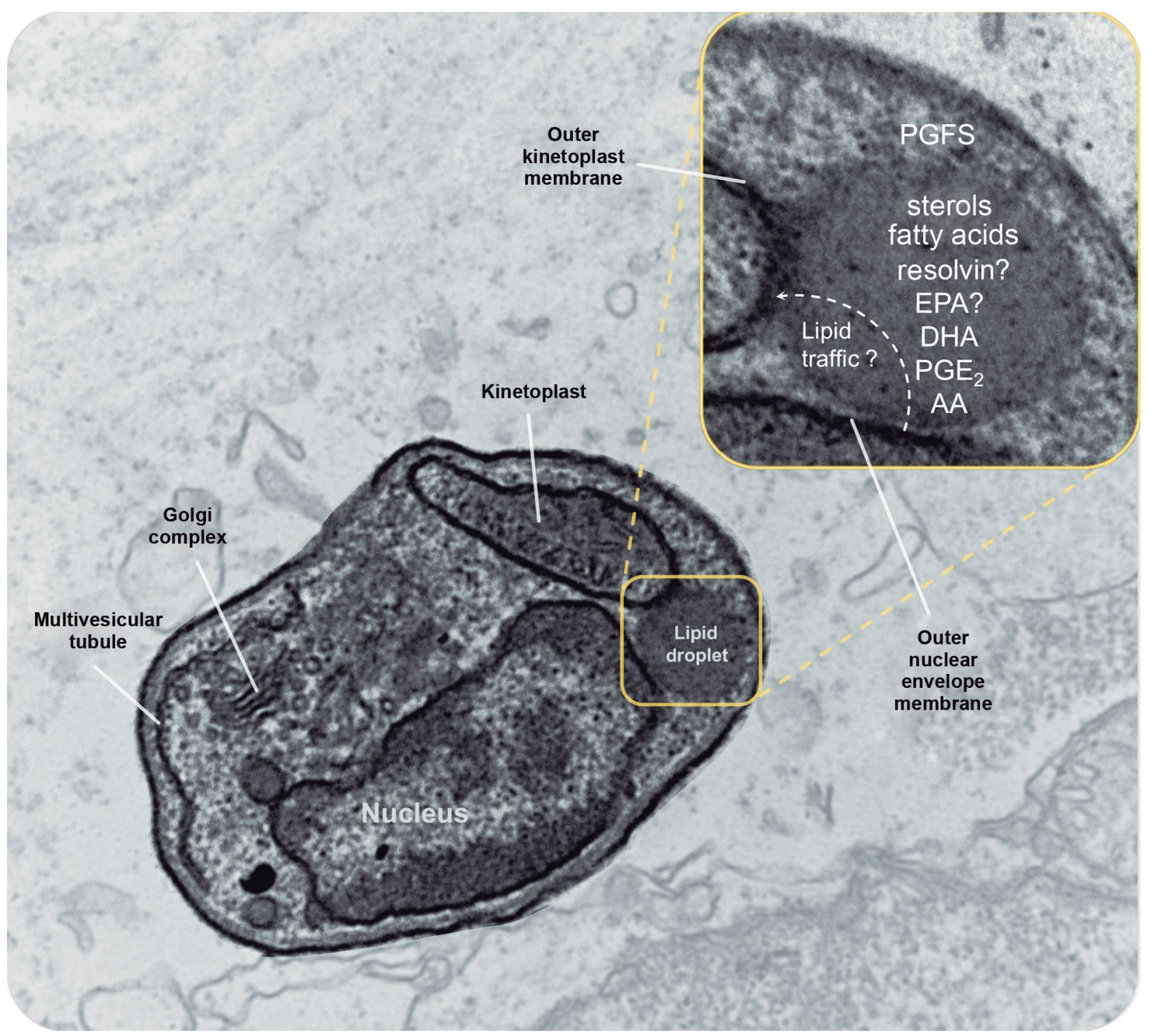

Schematic representation of interaction between lipid droplet (LD) and protozoan organelles. Schematic drawing on transmission electron microscopy illustrating the interaction lipid droplets, outer nuclear envelop membrane, and kinetoplast. Lipid droplet is responsible to storage and metabolise cholesteryl ester, fatty acids, ${ }^{(10,24,27)}$ and lipid mediators and their precursors, such as PGE $_{2}{ }^{(24)}$ but the presence of specialised lipids such as resolvins, and their precursors are still uncertain. In addition, LDs participate from the lipid traffic between intracellular organelles. Note the proximity of the outer nuclear envelop membrane and outer kinetoplast membranes to the LD hemi-membrane. TEM from Leishmania amastigote. Toxoplasma gondii and Trypanosoma cruzi also present LD interactions with mitochondria and endoplasmic reticulum. ${ }^{(10,15)}$

The cell death process also triggers the production of LDs in parasites. ${ }^{(18)}$ Antiparasitic drugs act by interfering with lipid metabolism or inhibiting mitochondrial activity, concomitantly inducing LD formation in trypanosomatids. ${ }^{(19)}$ Treatment with amiodarone in Leishmania amazonensis was found to lead to degenerative changes mainly in the structure, membrane, and function of mitochondria. This resulted in cell death marked by a dose-dependent accumulation of LDs, which were near autophagosomes and multivesicular bodies suggesting the induction of LD biogenesis as a result of neutral lipid storage from membrane degradation. ${ }^{(20)}$

Cells infected by protozoan parasites have their lipid metabolism and LD production altered in response to parasite-derived stimuli. When infecting hosts, parasites such as T. gondii and Leishmania major increase lipid accumulation inside the LDs of host cells and employ them in their own metabolism..$^{(11,21,22)}$ Further, T. gondii can interfere in the distribution of host organelles, such as mitochondria and LDs, by attracting them to parasitophorous vacuoles to access the lipids and incorporate them into their own LDs. Such strategies are vital for the survival inside the host, allowing them to recruit more resources for their lipid stores and metabolic processes. $(13,23)$ Furthermore, LDs of Trypanosoma cruzi trypomastigotes present large amounts of prostaglandin E2 $\left(\mathrm{PGE}_{2}\right)$, an important immunomodulatory, ${ }^{(24)}$ showing that demands arising from inflammatory processes also trigger LD biogenesis, which likely supports the parasite's survival during the course of infection. 


\section{Protozoan parasite LDs and lipid storage}

LDs are dynamic organelles that interact with several other cellular organelles; they are the storage and synthesis site of lipids, including important inflammatory mediators, in pathogenic protozoa. ${ }^{(25,26)}$ Epimastigotes of T. cruzi have LDs predominantly composed of neutral lipids, mainly cholesterol esters and there are indications that these organelles function as storage sites for exogenous cholesterol, mainly in the form of cholesterol esters. ${ }^{(15)}$ In addition, T. cruzi LDs have acylglycerols and, among bioactive lipids, polyunsaturated fatty acids, such as arachidonic acid (AA) and docosahexaenoic acid (DHA), ${ }^{(24)}$ are part of the fraction of free FAs present in the LDs of this parasite ${ }^{(15)}$ (Figure).

The formation of LDs can be modulated by lipids exogenous to protozoa. In Leishmania infantum and $T$. cruzi, exogenous AA induces the formation of LDs, as well as the formation of eicosanoids. ${ }^{(24,27)}$ Stimulating procyclic forms of $T$. brucei with oleic acid (OA) induces an increase in the number of LDs, and therefore, the synthesis and storage of TAGs in LDs of the protozoan. ${ }^{(28)}$ Supplementation of unsaturated FAs in T. gondii cells results in extremely large LDs and impairs protozoan replication, while saturated FAs do not lead to these effects. ${ }^{(29)}$ Furthermore, toxoplasma is unable to use FAs as a source for ATP synthesis, and both exposure to exogenous unsaturated FAs and inhibition of TgDGAT lead to excess lipids in the cytoplasm and consequent lipotoxicity due to alteration of intracellular membranes and, ultimately, to the death of the protozoan. ${ }^{(29)}$

In some protozoans, the increase in LDs is related to the differentiation process. Leishmania presents an increase in the number of LDs during the process of metacyclogenesis and amastigogenesis, presenting more LDs in the infectious forms. ${ }^{(27)} P$. falciparum mobilises lipids from LDs, serving as a source of precursors for the generation of membranes and replication of the protozoan. (30) LDs of $P$. falciparum can be found by performing physical interactions with the Plasmodium food vacuole $(\mathrm{VF}){ }^{(30)}$ Since neutral lipids facilitate the formation of $\beta$-hematin, ${ }^{(31,32)}$ the physical interaction between VF and LD could be responsible for supplying these lipids to the parasite, so that hematin crystallisation occurs more efficiently. In fact, not only has the formation of hemozoin crystals on the surface of LDs been documented, but also the possibility that the diameter of the organelle controls the size of these crystals. ${ }^{(32)}$ Therefore, LD may serve as a place for the formation of $\beta$-hematin, but further studies are needed to test this hypothesis.

\section{Lipid droplets protect protozoan parasites against oxidative stress}

The increase in LDs is a defense mechanism of pathogens against external aggressions arising from the host's immune system or from antiparasitic drugs. Some studies suggest that the change in the lipid profile of parasites may be a resistance mechanism, since strains of resistant parasites show changes in their lipid profiles when compared to susceptible strains. ${ }^{(33)}$ Therefore, LDs may be intrinsically related to the parasite's defense mechanisms, as LDs are the sites of lipid synthesis and storage.
A factor closely related to the maintenance of parasitic life is mitochondrial activity, which can be altered by an imbalance in the production of free radicals, as well as other reactive species. Trypanosomatids present detoxification mechanisms of reactive oxygen species, ${ }^{(34,35,36,37,38)}$ which involves at least two enzymes from the superoxide anion degradation pathway - the superoxide dismutase $1^{(39)}$ and trypanothione reductase enzymes ${ }^{(37)}$ being the two best-described parasitic enzymes. Antiparasitic drugs usually cause mitochondrial damage leading to the death of the parasite with an intracellular accumulation of lipids in LDs. ${ }^{(19)}$ Clomipramine induces cell death by inducing oxidative stress, which occurs concurrently with an increase in the number of LDs and peroxided lipids in L. amazonensis. The effects of clomipramine are reversed in the presence of antioxidants, such as $\mathrm{N}$ acetylcysteine (NAC), suggesting that the number of LDs increases in L. amazonensis to store peroxided lipids, protecting the cell from damage. ${ }^{(19)}$ A similar defense mechanism occurs during oxidative stress induced by miltefosine in strains of Leishmania donovani, in which drug-resistant parasites increase the expression of genes related to antioxidant mechanisms. ${ }^{(40)}$

In addition to the accumulation of oxidised lipids, LDs are the main sterol storage sites ${ }^{(15,41)}$ and the production of steroids can be a defense mechanism of protozoa, such as $L$. donovani, against oxidative stress generated by reactive species. Drugs with steroid biosynthesis inhibitory activity have an antiparasitic effect. However, inhibition of sterol synthesis alone is not able to suppress L. donovani intracellular survival. ${ }^{(42)}$ The inhibition of steroid synthesis can have an adjuvant action when combined with drugs that potentiate oxidative stress, such as antimony derivatives, since the combined use of these drugs increases the intracellular death of $L$. donovani. (42) It has even been shown that antioxidant substances, such as vitamin E, are produced by protozoa, such as $P$. falciparum, to protect them from oxidative stress generated by drugs and the host's defense mechanisms. ${ }^{(43,44)}$

In addition, studies have shown that prostaglandins are potential inducers of intracellular stress via reactive species production. $\mathrm{J}$ series metabolites, such as 15 -Deoxy-delta $^{(12,14)}$-prostaglandin $\mathrm{J} 2\left(15 \mathrm{~d}-\mathrm{PGJ}_{2}\right)$ and prostaglandin $\mathrm{D} 2\left(\mathrm{PGD}_{2}\right)$ are important bioactive lipid mediators capable of inducing an increase in reactive species in L. donovani and T. brucei promastigotes. However, further studies are needed to demonstrate LD involvement in a mechanism protecting against mitochondrial damage and death triggered by eicosanoid activation. ${ }^{(45,46)}$

\section{Protozoan parasites LDs in the metabolism of bioactive lipids}

There are still little data on the metabolism of eicosanoids in pathogenic protozoa, and most studies focus on parasites of the genera Trypanosoma and Leishmania ${ }^{(24,27,33,47-53)}$ (Table II). A variety of specialised lipid mediators and eicosanoids have been described in protozoa, but studies on the role of these mediators in the biology of the parasites, as well as in the parasite-host interaction, are still lacking. To date, studies have demonstrated the presence of resolvins (Rvs) ${ }^{(48)}$ eicosapentaenoic acid 


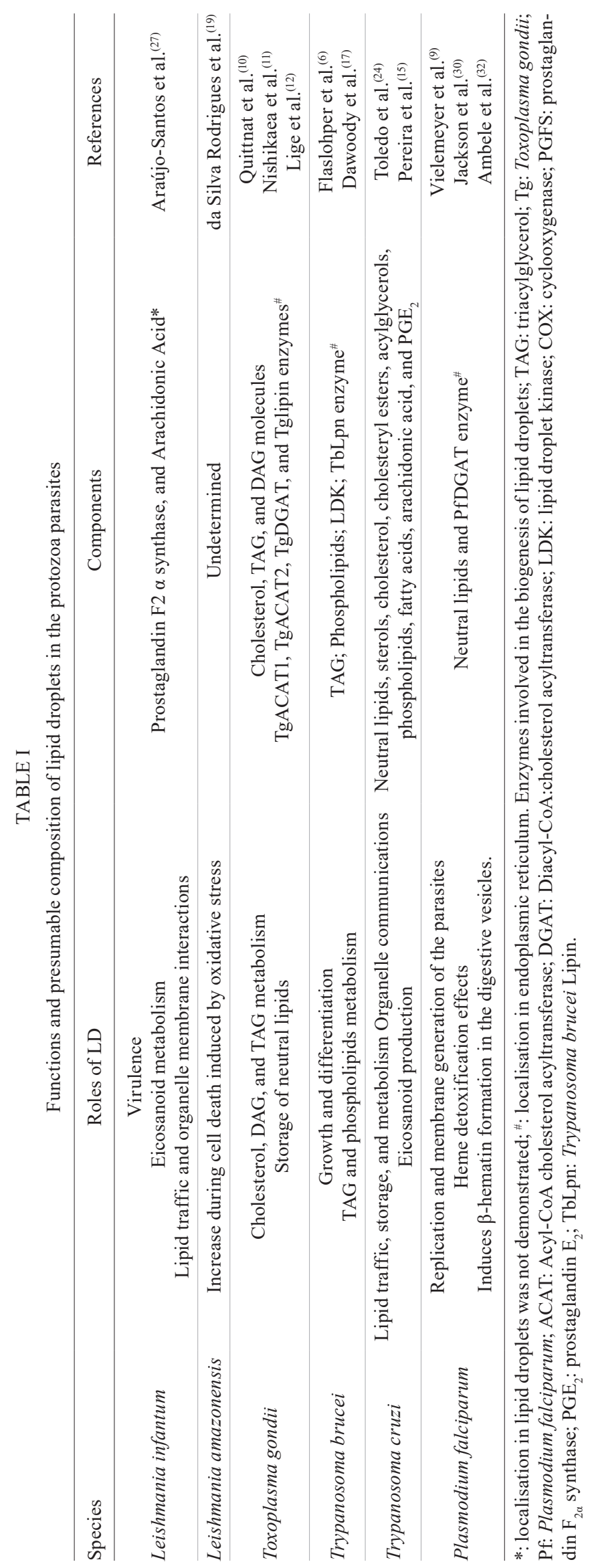



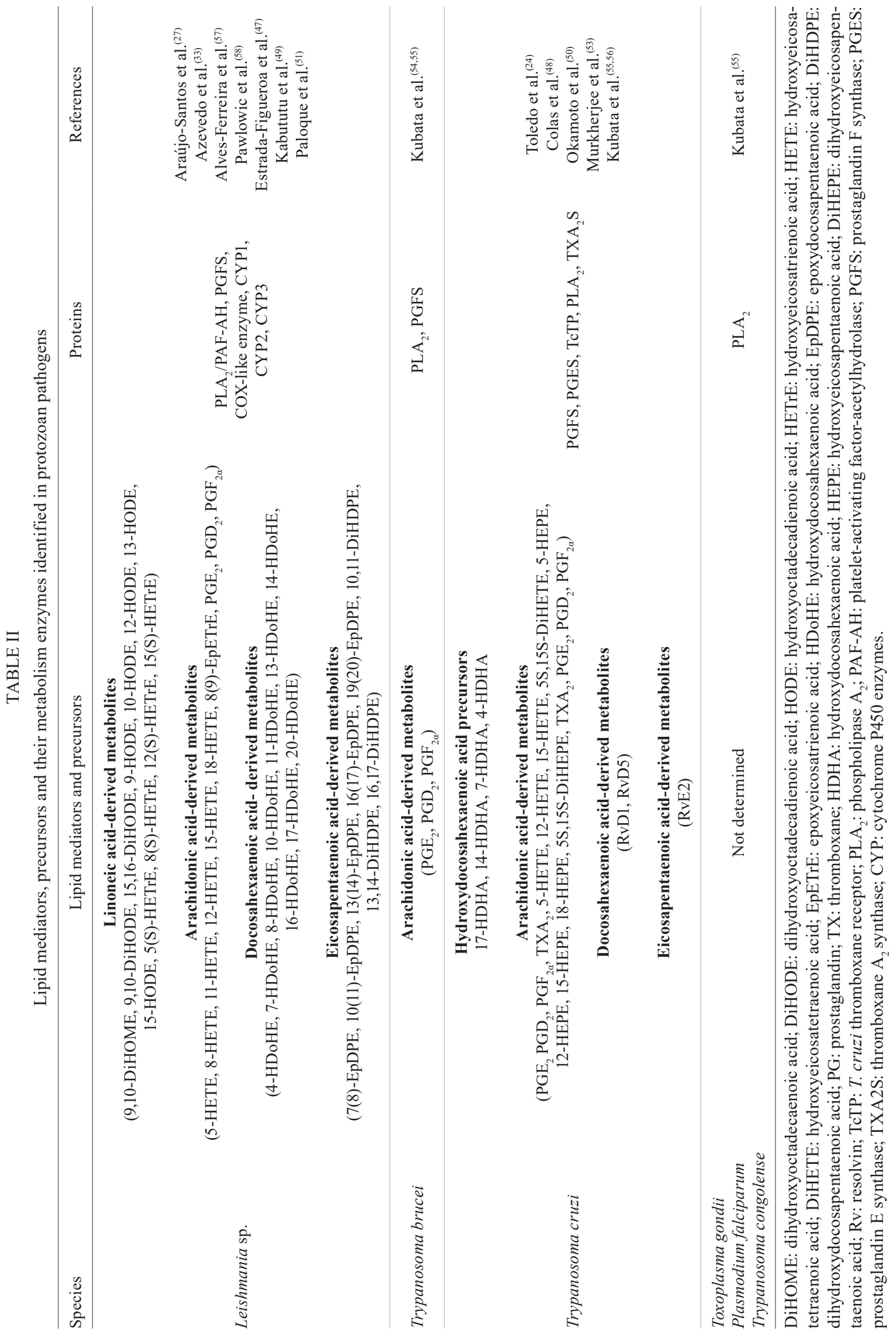
(EPA), AA, docosahexapentaenoic acid (DHA), ${ }^{(33,48)}$ prostaglandins (PGs) ${ }^{(27,48,49,51,54-58)}$ and thromboxanes $(\mathrm{TXs})^{(52)}$ in lipid extracts of parasites (Table II).

Parasites possess the necessary machinery for the synthesis of lipid mediators. ${ }^{(55)}$ Leishmania can produce PGs from PG synthases of their own LDs. ${ }^{(24,27)}$ Recently, it was discovered that the gp63 found in Leishmania mexicana is responsible for cyclooxygenase (COX)-like activity, emphasising the importance of eicosanoids and other lipid mediators capable of being synthesised by the parasite itself. ${ }^{(47)}$ In addition to COX, trypanosomatids have enzymes capable of synthesising other eicosanoids, such as $\mathrm{PGE}_{2}, \mathrm{PGD}_{2}$ and $\mathrm{PGF}_{2 \alpha}{ }^{(54,56)}$ T. cruzi trypomastigotes respond to exogenous $\mathrm{AA}$ stimulation with an increase in PGE synthase expression. ${ }^{(24)}$ The role of inflammatory lipids produced by the parasite during infection remains to be clarified. L. infantum LDs are capable of synthesising $\mathrm{PGF}_{2 \alpha}$, and this mediator is responsible for increasing the parasite's viability in the initial moments of infection by a mechanism yet to be determined. (27) Concomitantly, T. cruzi trypomastigotes synthesise RvD1 contributing to the resolution of the inflammatory process. ${ }^{(48)} T$. cruzi is also capable of synthesising and releasing TXs, a primarily pro-inflammatory molecule, which, however, starts to show anti-inflammatory activity due to the suppression of pathways in infection in vivo. ${ }^{(52)}$ In this sense, the presence of a thromboxane receptor has been demonstrated in T. cruzi, but its role in the biology of the pathogen is still uncertain. ${ }^{(53)}$ Another pathway for the production of lipid mediators has been described in parasites. ${ }^{(51)}$ Paloque at al. described cytochrome p450-like (CYP450) proteins in the L. infantum genome as responsible for producing polyunsaturated FA metabolites. CYP450-like proteins from L. infantum seem to be responsible for lipid precursor production of specialised lipids in this parasite. ${ }^{(51)}$ Thus, L. infantum is capable of releasing eicosanoids and other lipid mediators, but how these mediators alter the course of infection has not been established.

\section{CONCLUSIONS AND PERSPECTIVES}

The lipid droplets of different pathogenic protozoa are not a simply static lipid storage sites. These organelles are dynamic sites of lipid storages, and they are especially important for the parasite to function in the context of interaction with the cell host. LDs display a central importance in the growth, differentiation, infectivity, and lipid metabolism of protozoan parasites (Table I). Proteomic and lipidomic studies on isolation of protozoan lipid droplets are still needed to establish the functions performed by these organelles. Furthermore, comparative genomic studies can be conducted to transpose the knowledge of different pathogenic protozoa to identify the genes responsible for the biogenesis and elimination of LDs. An understanding of lipid metabolism, especially of bioactive lipids, and the role of protozoan LDs in this context, may help to identify potential targets for the development of antiparasitic drugs. Therefore, LDs are promising research targets for future methods of controlling infection based on the cell biology of pathogenic protozoa.

\section{AUTHORS' CONTRIBUTION}

VST, MVC, RSOS, JB-L, IKGA, VMB and TA-S wrote different sections, edited, and reviewed the manuscript. The authors declare that the research was conducted in the absence of any commercial or financial relationships that could be construed as a potential conflict of interest.

\section{REFERENCES}

1. Tauchi-Sato K, Ozeki S, Houjou T, Taguchi R, Fujimoto T. The surface of lipid droplets is a phospholipid monolayer with a unique fatty acid composition. J Biol Chem. 2002; 277(46): 44507-12.

2. Bozza PT, Bandeira-Melo C. Mechanisms of leukocyte lipid body formation and function in inflammation. Mem Inst Oswaldo Cruz. 2005; 100(Suppl. 1): 113-20.

3. Welte MA, Gould AP. Lipid droplet functions beyond energy storage. Biochim Biophys Acta Mol Cell Biol Lipids. 2017; 1862(10 Pt B): $1260-72$.

4. Greenberg AS, Egan JJ, Wek SA, Garty NB, Blanchette-Mackie EJ, Londos C. Perilipin, a major hormonally regulated adipocytespecific phosphoprotein associated with the periphery of lipid storage droplets. J Biol Chem. 1991; 266(17): 11341-6.

5. Martin S, Parton RG. Lipid droplets: a unified view of a dynamic organelle. Nat Rev Mol Cell Biol. 2006; 7: 373-8.

6. Flaspohler JA, Jensen BC, Saveria T, Kifer CT, Parsons M. A novel protein kinase localized to lipid droplets is required for droplet biogenesis in trypanosomes. Eukaryot Cell. 2010; 9(11): 1702-10.

7. Farese RV, Walther TC. Lipid droplets finally get a little R-E-S-PE-C-T. Cell. 2009; 139(5): 855-60.

8. Barbosa AD, Savage DB, Siniossoglou S. Lipid droplet-organelle interactions: emerging roles in lipid metabolism. Curr Opin Cell Biol. 2015; 35: 91-7.

9. Vielemeyer O, McIntosh MT, Joiner KA, Coppens I. Neutral lipid synthesis and storage in the intraerythrocytic stages of Plasmodium falciparum. Mol Biochem Parasitol. 2004; 135(2): 197-209.

10. Quittnat F, Nishikawa Y, Stedman TT, Voelker DR, Choi J-Y, Zahn MM, et al. On the biogenesis of lipid bodies in ancient eukaryotes: synthesis of triacylglycerols by a Toxoplasma DGAT1related enzyme. Mol Biochem Parasitol. 2004; 138(1): 107-22.

11. Nishikawa Y, Quittnat F, Stedman TT, Voelker DR, Choi J-Y, Zahn M, et al. Host cell lipids control cholesteryl ester synthesis and storage in intracellular Toxoplasma. Cell Microbiol. 2005; 7(6): 849-67.

12. Lige B, Sampels V, Coppens I. Characterization of a second sterol-esterifying enzyme in Toxoplasma highlights the importance of cholesterol storage pathways for the parasite. Mol Microbiol. 2013; 87(5): 951-67.

13. Nolan SJ, Romano JD, Coppens I. Host lipid droplets: an important source of lipids salvaged by the intracellular parasite Toxoplasma gondii. PLoS Pathog. 2017; 13(6): e1006362.

14. Dass S, Shunmugam S, Berry L, Arnold C-S, Katris NJ, Duley $\mathrm{S}$, et al. Toxoplasma LIPIN is essential in channeling host lipid fluxes through membrane biogenesis and lipid storage. Nat Commun. 2021; 12(1): 2813.

15. Pereira MG, Visbal G, Costa TFR, Frases S, de Souza W, Atella G, et al. Trypanosoma cruzi epimastigotes store cholesteryl esters in lipid droplets after cholesterol endocytosis. Mol Biochem Parasitol. 2018; 224(3): 6-16.

16. Rambold AS, Cohen S, Lippincott-Schwartz J. Fatty acid trafficking in starved cells: regulation by lipid droplet lipolysis, autophagy, and mitochondrial fusion dynamics. Dev Cell. 2015; 32(6): 678-92. 
17. Nejad LD, Serricchio M, Jelk J, Hemphill A, Bütikofer P. TbLpn, a key enzyme in lipid droplet formation and phospholipid metabolism, is essential for mitochondrial integrity and growth of Trypanosoma brucei: role of lipin in T. brucei growth. Mol Microbiol. 2018; 109(1): 105-20

18. Mendes EA, Desoti VC, Silva SO, Ueda-Nakamura T, Dias Filho BP, Yamada-Ogatta SF, et al. C5 induces different cell death pathways in promastigotes of Leishmania amazonensis. Chem Biol Int. 2016; 256: 16-24

19. Rodrigues JHS, Miranda N, Volpato H, Ueda-Nakamura T, Nakamura $\mathrm{CV}$. The antidepressant clomipramine induces programmed cell death in Leishmania amazonensis through a mitochondrial pathway. Parasitol Res. 2019; 118(3): 977-89.

20. de Macedo-Silva ST, Silva TLAO, Urbina JA, de Souza W, Rodrigues JCF. Antiproliferative, ultrastructural, and physiological effects of amiodarone on promastigote and amastigote forms of Leishmania amazonensis. Mol Biol Int. 2011; 2011: 876021.

21. Rabhi S, Rabhi I, Trentin B, Piquemal D, Regnault B, Goyard S, et al. Lipid droplet formation, their localization and dynamics during Leishmania major macrophage infection. PLoS One. 2016; 11(2): $\mathrm{e} 0148640$.

22. Gomes AF, Magalhães KG, Rodrigues RM, de Carvalho L, Molinaro R, Bozza PT, et al. Toxoplasma gondii-skeletal muscle cells interaction increases lipid droplet biogenesis and positively modulates the production of IL-12, IFN-g and PGE2. Parasit Vectors. 2014; 7(1): 47.

23. Charron AJ, Sibley LD. Host cells: mobilizable lipid resources for the intracellular parasite Toxoplasma gondii. J Cell Sci. 2002; 115(Pt 15): 3049-59.

24. Toledo DAM, Roque NR, Teixeira L, Milán-Garcés EA, Carneiro $\mathrm{AB}$, Almeida MR, et al. Lipid body organelles within the parasite Trypanosoma cruzi: a role for intracellular arachidonic acid metabolism. PLoS One. 2016; 11(8): e0160433.

25. Gao Q, Goodman JM. The lipid droplet - a well-connected organelle. Front Cell Dev Biol. 2015; 3(8): 1-12.

26. de Almeida PE, Toledo DAM, Rodrigues GSC, D’Avila H. Lipid bodies as sites of prostaglandin E2 synthesis during Chagas disease: impact in the parasite escape mechanism. Front Microbiol. 2018; 9: 499

27. Araújo-Santos T, Rodríguez NE, Moura-Pontes S, Dixt UG, Abánades DR, Bozza PT, et al. Role of prostaglandin F2 $\alpha$ production in lipid bodies from Leishmania infantum chagasi: insights on virulence. J Infect Dis. 2014; 210(12): 1951-61.

28. Allmann S, Mazet M, Ziebart N, Bouyssou G, Fouillen L, Dupuy $\mathrm{J}-\mathrm{W}$, et al. Triacylglycerol storage in lipid droplets in procyclic Trypanosoma brucei. PLoS One. 2014; 9(12): el14628.

29. Nolan SJ, Romano JD, Kline JT, Coppens I. Novel approaches to dill Toxoplasma gondii by exploiting the uncontrolled uptake of unsaturated fatty acids and vulnerability to lipid storage inhibition of the parasite. Antimicrob Agents Chemother. 2018; 62(10): e00347-18

30. Jackson KE, Klonis N, Ferguson DJP, Adisa A, Dogovski C, Tilley L. Food vacuole-associated lipid bodies and heterogeneous lipid environments in the malaria parasite, Plasmodium falciparum. Mol Microbiol. 2004; 54(1): 109-22.

31. Pandey AV, Babbarwal VK, Okoyeh JN, Joshi RM, Puri SK, Singh RL, et al. Hemozoin formation in malaria: a two-step process involving histidine-rich proteins and lipids. Biochem Biophys Res Commun. 2003; 308(4): 736-43.

32. Ambele MA, Sewell BT, Cummings FR, Smith PJ, Egan TJ. Syn- thetic Hemozoin ( $\beta$-Hematin) crystals nucleate at the surface of neutral lipid droplets that control their sizes. Cryst Growth Des. 2013; 13(10): 4442-52.

33. De Azevedo AF, Dutra JLL, Santos MLB, Santos DA, Alves PB, De Moura TR, et al. Fatty acid profiles in Leishmania spp. isolates with natural resistance to nitric oxide and trivalent antimony. $\mathrm{Pa}-$ rasitol Res. 2014; 113(1): 19-27.

34. Freire ACG, Alves CL, Goes GR, Resende BC, Moretti NS, Nunes VS, et al. Catalase expression impairs oxidative stressmediated signalling in Trypanosoma cruzi. Parasitology. 2017; 144(11): 1498-510

35. Tavares NM, Araújo-Santos T, Afonso L, Nogueira PM, Lopes UG, Soares RP, et al. Understanding the mechanisms controlling Leishmania amazonensis infection in vitro: the role of LTB4 derived from human neutrophils. J Infect Dis. 2014; 210(4): 656-66.

36. Machado-Silva A, Cerqueira PG, Grazielle-Silva V, Gadelha FR, Peloso EF, Teixeira SMR, et al. How Trypanosoma cruzi deals with oxidative stress: antioxidant defense and DNA repair pathways. Mutat Res Rev Mutat Res. 2016; 767: 8-22.

37. Ghosh AK, Saini S, Das S, Mandal A, Sardar AH, Ansari MY, et al. Glucose-6-phosphate dehydrogenase and Trypanothione reductase interaction protects Leishmania donovani from metalloid mediated oxidative stress. Free Radic Biol Med. 2017; 106: 10-23.

38. Singh K, Ali V, Singh KP, Gupta P, Suman SS, Ghosh AK, et al. Deciphering the interplay between cysteine synthase and thiol cascade proteins in modulating Amphotericin B resistance and survival of Leishmania donovani under oxidative stress. Redox Biol. 2017; 12: 350-66.

39. Khouri R, Novais F, Santana G, de Oliveira CI, dos Santos MAV, Barral A, et al. DETC induces Leishmania parasite killing in human in vitro and murine in vivo models: a promising therapeutic alternative in leishmaniasis. PLoS One. 2010; 5(12): 8-9.

40. Deep DK, Singh R, Bhandari V, Verma A, Sharma V, Wajid S, et al. Increased miltefosine tolerance in clinical isolates of Leishmania donovani is associated with reduced drug accumulation, increased infectivity and resistance to oxidative stress. PLoS Negl Trop Dis. 2017; 11(6): e0005641.

41. Walther TC, Farese RVJ. Lipid droplets and cellular lipid metabolism. Annu Rev Biochem. 2012; 81: 687-714.

42. Mathur R, Das RP, Ranjan A, Shaha C. Elevated ergosterol protects Leishmania parasites against antimony-generated stress. FASEB J. 2015; 29(10): 4201-13.

43. Sussmann RAC, Fotoran WL, Kimura EA, Katzin AM. Plasmodium falciparum uses vitamin E to avoid oxidative stress. Parasit Vectors. 2017; 10(1):461.

44. Sussmann RAC, Angeli CB, Peres VJ, Kimura EA, Katzin AM. Intraerythrocytic stages of Plasmodium falciparum biosynthesize vitamin E. FEBS Lett. 2011; 585(24): 3985-91.

45. Vishwakarma P, Parmar N, Yadav PK, Chandrakar P, Kar S. 15d-Prostaglandin $\mathrm{J} 2$ induced reactive oxygen species-mediated apoptosis during experimental visceral leishmaniasis. J Mol Med. 2016; 94(6): 695-710.

46. Figarella K, Uzcategui NL, Beck A, Schoenfeld C, Kubata BK, Lang F, et al. Prostaglandin-induced programmed cell death in Trypanosoma brucei involves oxidative stress. Cell Death Differ. 2006; 13(10): 1802-14.

47. Estrada-Figueroa LA, Díaz-Gandarilla JA, Hernández-Ramírez VI, Arrieta-González MM, Osorio-Trujillo C, Rosales-Encina JL, et al. Leishmania mexicana gp63 is the enzyme responsible for cyclooxygenase (COX) activity in this parasitic protozoa. Biochimie. 2018; 151: 73-84. 
48. Colas RA, Ashton AW, Mukherjee S, Dalli J, Akide-Ndunge OB, Huang H, et al. Trypanosoma cruzi produces the specialized proresolving mediators resolvin D1, resolvin D5, and resolvin E2. Infect Immun. 2018; 86(4): 1-10.

49. Kabututu Z, Martin SK, Nozaki T, Kawazu S, Okada T, Munday $\mathrm{CJ}$, et al. Prostaglandin production from arachidonic acid and evidence for a 9,11-endoperoxide prostaglandin $\mathrm{H} 2$ reductase in Leishmania. Int J Parasitol. 2003; 33(2): 221-8.

50. Okamoto N, Yamaguchi K, Mizohata E, Tokuoka K, Uchiyama $\mathrm{N}$, Sugiyama S, et al. Structural insight into the stereoselective production of PGF $2 \alpha$ by old yellow enzyme from Trypanosoma cruzi. J Biochem (Tokyo). 2011; 150(5): 563-8.

51. Paloque L, Perez-Berezo T, Abot A, Dalloux-Chioccioli J, Bourgeade-Delmas S, Le Faouder P, et al. Polyunsaturated fatty acid metabolites: biosynthesis in Leishmania and role in parasite/ host interaction. J Lipid Res. 2019; 60(3): 636-47.

52. Ashton AW, Mukherjee S, Nagajyothi FNU, Huang H, Braunstein VL, Desruisseaux MS, et al. Thromboxane $\mathrm{A}_{2}$ is a key regulator of pathogenesis during Trypanosoma cruzi infection. J Exp Med. 2007; 204(4): 929-40.
53. Mukherjee S, Sadekar N, Ashton AW, Huang H, Spray DC, Lisanti MP, et al. Identification of a functional prostanoid-like receptor in the protozoan parasite, Trypanosoma cruzi. Parasitol Res. 2013; 112(4): $1417-25$.

54. Kubata BK, Duszenko M, Kabututu Z, Rawer M, Szallies A, Fujimori K, et al. Identification of a novel prostaglandin $\mathrm{f}(2 \mathrm{alpha})$ synthase in Trypanosoma brucei. J Exp Med. 2000; 192(9): 1327-38.

55. Kubata BK, Duszenko M, Martin KS, Urade Y. Molecular basis for prostaglandin production in hosts and parasites. Trends Parasitol. 2007; 23(7): 325-31

56. Kubata BK, Kabututu Z, Nozaki T, Munday CJ, Fukuzumi S, Ohkubo $\mathrm{K}$, et al. A key role for old yellow enzyme in the metabolism of drugs by Trypanosoma cruzi. J Exp Med. 2002; 196(9): 1241-51.

57. Alves-Ferreira EVC, Ferreira TR, Walrad P, Kaye PM, Cruz AK. Leishmania braziliensis prostaglandin $\mathrm{F} 2 \alpha$ synthase impacts host infection. Parasit Vectors. 2020; 13(1): 9.

58. Pawlowic MC, Zhang K. Leishmania parasites possess a plateletactivating factor acetylhydrolase important for virulence. Mol Biochem Parasitol. 2012; 186(1): 11-20. 